\title{
Optimization of 3D EBSD in a FIB-SEM System Using a Static Sample Setup
}

Julien Guyon ${ }^{1,2}$, Nathalie Gey ${ }^{1,2}$, Daniel Goran ${ }^{3}$, Smail Chalal ${ }^{4}$, and Fabián Pérez-Willard ${ }^{4}$.

1. Laboratoire d’Etude des Microstructures et de Mécanique des Matériaux, LEM3, CNRS ISGMP, Université de Lorraine, F-57045 Metz Cedex 01, France

2. Laboratory of Excellence on Design of Alloy Metals for low-mAss Structures ('LabEx DAMAS'), Université de Lorraine, France

3. Bruker Nano GmbH, Am Studio 2D, 12489 Berlin, Germany

4. Carl Zeiss Microscopy GmbH, Carl-Zeiss Str. 56, 73447 Oberkochen, Germany

Recently, the need for a comprehensive characterization of grains and grain boundaries has led to the extension of electron backscatter diffraction (EBSD) into three dimensions (3D). 3D EBSD can be performed by serial sectioning the sample with robot assisted grinding and polishing [1], laser ablation [2], or focused ion beam (FIB) milling [3] in automated processes.

In this note we report about an optimized experimental setup for 3D EBSD data acquisition in a conventional FIB-SEM instrument. In contrast to other 3D EBSD experiments in a FIB-SEM, in our setup the sample remains static during the entire experiment. The advantages in terms of data quality and throughput are discussed and illustrated with measurements on a coarse-grained INCONEL 718 nickel-based superalloy as a model material.

Experimental Procedures:

The static 3D EBSD setup was realized on a customized ZEISS Auriga 40 FIB-SEM instrument equipped with a high resolution Bruker e-Flash EBSD detector. The original chamber of the ZEISS Auriga 40 was redesigned to accommodate the EBSD camera at an azimuthal angle $\varphi=-15.3^{\circ}$ (see Fig. 1(a) ). The exact value of $\varphi$ results from geometrical considerations, namely: a) FIB and SEM beam are at an angle of $54^{\circ}$ for the Auriga 40; b) the FIB beam is parallel to the FIB prepared surface, which is c) tilted $70^{\circ}$ from the horizontal [4]. The EBSD camera can be positioned as close as $15 \mathrm{~mm}$ to the FIBSEM coincidence point, which is at a working distance of $5 \mathrm{~mm}$. Using the Application Programming Interfaces (API) of FIB-SEM and EBSD systems the 3D EBSD acquisition workflow was fully automated.

An INCONEL 718 sample, roughly $(10 \times 10 \times 5) \mathrm{mm}^{3}$ in size, was used to characterize the experimental setup and evaluate the resulting 3D-data quality. The nickel-based superalloy is well suited for this purpose, because it yields high-quality EBSD patterns on $30 \mathrm{kV}$ FIB polished surfaces even at a high EBSD acquisition speed of $140 \mathrm{~Hz}$. Moreover the recrystallized grains contain numerous coherent twins. Their well known $\{111\}$ boundary planes are used as a reference to quantify raw data alignment.

Main Results:

Usually, in a FIB-SEM the sample is moved iteratively between milling and EBSD positions during the 3D EBSD experiment. Such stage movements - and corresponding stage settling times - are eliminated in the static setup. Additionally, no time is spent on image registration steps after each stage movement, which are needed to reposition accurately FIB and SEM beams relative to the volume of interest (VOI) 
if the sample is moved. Thus, 3D EBSD acquisition is simpler and the analyzed volume rate increased when performed under static conditions.

Figure 1(b) shows reconstructed 3D EBSD data using [5] for the INCONEL 718 sample. In the center of the VOI a twinned grain is observed. The VOI has been sectioned to show a reconstructed plane perpendicular to the original EBSD acquisition plane. This plane runs through the twinned grain. At this point, it is important to stress that neither drift correction operations were performed during the 3D EBSD run nor data post-processing after the 3D run to ensure alignment of the different sections to each other. Nonetheless the twin boundaries in the rendered surface appear almost perfectly flat, which demonstrates the impressive alignment quality of the raw 3D data. They correspond to a $\{111\}$ plane.

In the static setup it is conceivable to increase throughput further by performing the EBSD measurement while milling. Prerequisite is to be able to index the EBSD patterns despite the noise introduced by FIB. To explore this possibility, EBSD patterns of a selected grain were measured while milling simultaneously the sample with different FIB probe currents. Even for FIB currents up to $30 \mathrm{nA}$, the patterns were good enough to be indexed with high confidence.

\section{References:}

[1] M. Uchic et al, $1^{\text {st }}$ International Conference on 3D Materials Science (2012) p. 195-202

[2] M. P. Echlin et al, Acta Mater. 64 (2014), p. 307-315

[3] S. Zaefferer and S. I. Wright, in "Electron Backscatter Diffraction in Materials Science”, ed.

A. J. Schwartz et al (Springer 2009), p. 109-122

[4] D. Dönitz and Ch. Wagner, US Patent US8901510 B2 (2014)

[5] M. A. Groeber and M. A. Jackson, Integr. Mater. Manuf. Innov. (2014) 3:5
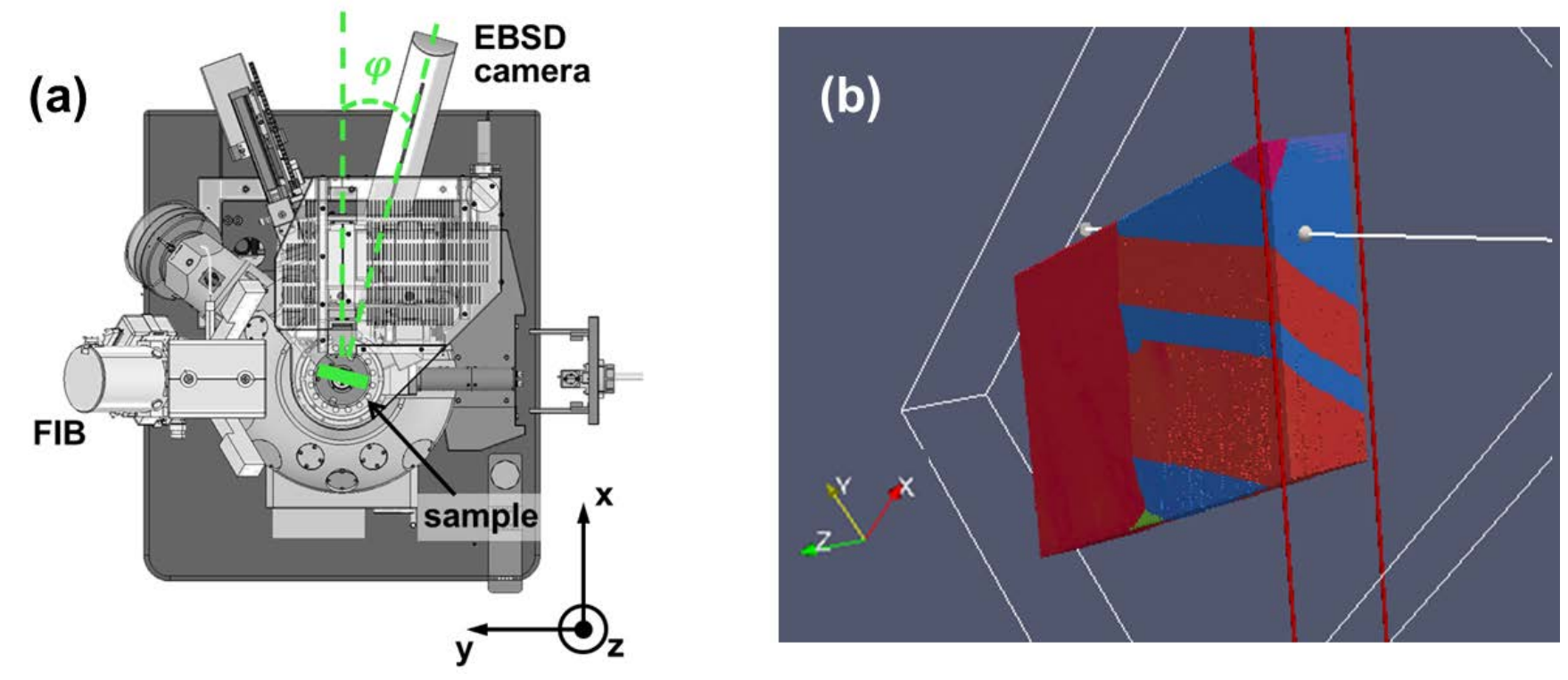

Figure 1. (a) Top view CAD drawing of the customized FIB-SEM instrument. The EBSD camera is positioned at an azimuthal angle $\varphi=-15.3^{\circ}$ to realize the optimal static 3D EBSD setup. (b) Reconstructed 3D EBSD volume of an INCONEL 718 sample. The volume has been sectioned along a plane perpendicular to the EBSD acquisition plane to show the alignment quality of the raw data. 